\title{
Pricing of an Exotic Forward Contract
}

\author{
Jirô Akahori, Yuji Hishida and Maho Nishida \\ Dept. of Mathematical Sciences, Ritsumeikan University \\ 1-1-1 Nojihigashi, Kusatsu, Shiga 525-8577, Japan \\ E-mail: \{akahori, rp003994,rs028990\}@se.ritsumei.ac.jp
}

\begin{abstract}
In this paper we study a pricing problem of an $e x$ otic Forward contract. Unlike the standard Forward, the contract is not fair, and like an option, it is compensated by the premium. Using standard arguments in the Black-Scholes economy, an explicit formula for hedging as well as pricing is obtained. This is possible because of an exotic way of settlement, which is another focus of this paper. Contrary to our intuition, simpler ways of settlement do not necessarily imply a simpler formula.
\end{abstract}

\section{Introduction}

A Forward contract is an agreement between two parties to buy or sell an asset (which can be of any kind) on a pre-agreed future day at a pre-agreed price which is called Forward price. Usually the Forward price is set to make the contract fair, and so no payment takes place on the agreement day. For details of standard Forward contracts, see e.g. [2].

If an extra agreement that benefits one party only is added to the contract, as is the situation that we will study, then an adjustment is needed to keep the contract fair. There are two possibilities: one is by a change of the Forward price, and the other is by payment at the agreement day.

In this paper we will study such a contract which we call exotic Forward. We adopt the latter adjustment and study the premium - how much should be paid for the contract.

The Exotic Forward we will study is defined by the following

Extra Agreement 1. A buyer has a right to choose the price-date at the delivery day between $N_{1}$ days before the delivery day and $N_{2}$ days after,

with the

Extra Agreement 2 (Settlement of Difference). A settlement day is the delivery day in both cases. If a buyer chooses the $N_{2}$ days after, the payment is taken by the way of settlement of difference; she/he is paid a pre-agreed price at the delivery day $T$. and then is paid the difference at $T+N_{2}$.
We will give an explicit formula for the premium and the corresponding hedging strategy under a BlackScholes type model. What interests us most is that the formula is very much alike the Black-Scholes formula for the plain European option. The reduction is caused by the exotic way of settlement (Extra Agreement 2).

\section{The Setting and Notations}

We denote the delivery day by $T, T_{1}:=T-N_{1}$, and $T_{2}=T+N_{2}$. We assume the Black-Scholes Economy; i.e., a spot price $S=\left\{S_{t}\right\}_{0<t<T_{2}}$ and risk-free bond $B=$ $\left\{B_{t}\right\}_{0 \leq t \leq T_{2}}$ satisfy the following stochastic differential equations:

$$
\begin{aligned}
d S_{t} & =\sigma S_{t} d W_{t}+\mu S_{t} d t \\
d B_{t} & =r B_{t} d t
\end{aligned}
$$

where $\sigma>0, r \geq 0, \mu \in \mathbb{R}$, and $W$ is a standard 1dimensional Brownian motion. For simplicity, we assume that $B_{0}=1$.

Under these settings, the Exotic Forward contract in question is restated as follows.

1. The agreement day is time 0 , when the Forward price $K$ is quoted in the market.

2. The buyer pays the premium $\pi$ on the agreement day.

3. On the delivery day $T$, the buyer chooses the price day; $T_{1}$ or $T_{2}$.

4. If she/he chooses $T_{1}$, she/he is paid $S_{T_{1}}-K$.

5. If she/he chooses $T_{2}$, she/he is paid $Y_{T}$ for the moment.

6. And then on $T_{2}$, she/he is paid the difference $S_{T_{2}}-K-Y_{T}$.

Though studying general $Y_{T}$ is possible, we concentrate on the case $Y_{T}=S_{T}-K$ in this paper.

Before stating the results, we introduce some notations. Let

$d_{ \pm}(t, x)=\frac{\left(r \pm \frac{1}{2} \sigma^{2}\right)(T-t)+\log \left(2-e^{-r\left(T_{2}-T\right)}\right) x / S_{T_{1}}}{\sigma \sqrt{T-t}}$ 
for $t \in\left[T_{1}, T\right)$ and $x>0$. We write simply $d_{ \pm}$for $d_{ \pm}\left(T_{1}, S_{T_{1}}\right)$. That is,

$$
d_{ \pm}=\frac{\left(r \pm \frac{1}{2} \sigma^{2}\right)\left(T-T_{1}\right)+\log \left(2-e^{-r\left(T_{2}-T\right)}\right)}{\sigma \sqrt{T-T_{1}}} .
$$

Let also

$$
A:=\left\{S_{T_{1}} \leq\left(2-e^{-r\left(T_{2}-T\right)}\right) S_{T}\right\} .
$$

Finally, we use the following conventions:

$$
\phi(x)=\frac{1}{\sqrt{2 \pi}} e^{-\frac{x^{2}}{2}}
$$

and

$$
\Phi(x)=\int_{-\infty}^{x} \phi(y) d y,
$$

the density and the distribution function of the standard Gaussian random variable.

\section{The Results}

The first result which corresponds to the pricing is the following.

Theorem 1. Under the hypothesis of No-Arbitrage, the fair premium is given by

$$
\begin{aligned}
\pi & =S_{0} e^{-r\left(T-T_{1}\right)} \Phi\left(-d_{-}\right) \\
& +S_{0}\left(2-e^{-r\left(T_{2}-T\right)}\right) \Phi\left(d_{+}\right)-e^{-r T} K .
\end{aligned}
$$

From the above result, we notice that the premium of this contract is represented by a mono-integral, even though this contract has several exotic aspects. We stress that when $Y_{T}=0$, i.e. no settlement of difference, the premium is no more mono-integral; a little far away from Black-Scholes formula.

Further, we can also obtain the explicit hedging strategy for the seller as follows.

Theorem 2. The contract is perfectly hedged by holding

$$
\eta_{t}= \begin{cases}e^{-r\left(T-T_{1}\right)} \Phi\left(-d_{-}\right) & \left(0 \leq t<T_{1}\right) \\ +\left(2-e^{-r\left(T_{2}-T\right)}\right) \Phi\left(d_{+}\right)=: \eta_{0}, & \\ \eta_{0}+\left\{\left(2-e^{-r\left(T_{2}-T\right)}\right) \phi\left(d_{+}\left(t, S_{t}\right)\right)\right. & \left(T_{1} \leq t<T\right) \\ \left.-\frac{B_{t} S_{T_{1}}}{B_{T} S_{t}} \phi\left(-d_{-}\left(t, S_{t}\right)\right)\right\} / \sigma \sqrt{T-t}, & \\ 1_{A} & \left(T \leq t<T_{2}\right)\end{cases}
$$

amount of the risky asset at time $t \in\left[0, T_{2}\right)$, and

$$
\nu_{t}=\left\{\begin{array}{cc}
-K / B_{T}=: \nu_{0}, & \left(0 \leq t<T_{1}\right) \\
\nu_{0}+\left(2-e^{r\left(T_{2}-T\right)}\right) \frac{S_{t} \phi\left(d_{+}\left(t, S_{t}\right)\right)}{B_{t}^{-1} \sigma \sqrt{T-t}} & \\
+S_{T_{1}} B_{T}^{-1}\left\{\Phi\left(-d_{-}\left(t, S_{t}\right)\right)\right. & \left(T_{1} \leq t<T\right) \\
\left.-\frac{\phi\left(-d_{-}\left(t, S_{t}\right)\right)}{\sigma \sqrt{T-t}}\right\}, & \\
\nu_{0}+1_{A} S_{T}\left(B_{T}^{-1}-B_{T_{2}}^{-1}\right) & \left(T \leq t<T_{2}\right) \\
+1_{\Omega \backslash A} S_{T_{1}} B_{T}^{-1} &
\end{array}\right.
$$

Remark. The hedging strategy is statistical unless $t \in$ $\left[T_{1}, T\right)$, as is the case with standard Forward contracts; known as cost of carry (see e.g. [2]). The exotic aspect of our contract forces the seller to hedge dynamically, though it is needed only for $t \in\left[T_{1}, T\right)$.

\section{No-Arbitrage Hypothesis}

Firstly, we review the consequences of the NoArbitrage hypothesis on the Black-Scholes economy (1).

Let $(\Omega, \mathcal{F}, \boldsymbol{P})$ be a probability space on which $W$ is defined, and $\left\{\mathcal{F}_{t}\right\}$ be the natural filtration of $W$. Put $\theta:=(\mu-r) / \sigma$. The $\boldsymbol{P}-\{\mathcal{F}\}$-martingale $Z_{t}:=\exp \left(\theta W_{t}-\right.$ $\left.\frac{1}{2} \theta^{2} t\right)$ defines an equivalent probability measure $\hat{\boldsymbol{P}}$ by

$$
\left.\frac{d \hat{\boldsymbol{P}}}{d \boldsymbol{P}}\right|_{\mathcal{F}_{t}}=Z_{t}, \quad t \in\left[0, T_{2}\right]
$$

The probability measure $\hat{\boldsymbol{P}}$ is usually called equivalent martingale measure, EMM for short, and plays a central role in the pricing of options and other derivatives.

The Black-Scholes economy (1) under the EMM is

$$
\begin{aligned}
d S_{t} & =\sigma S_{t} d \hat{W}_{t}+r S_{t} d t \\
d B_{t} & =r B_{t} d t
\end{aligned}
$$

or equivalently

$$
\begin{aligned}
& S_{t}=S_{0} \exp \left\{\sigma \hat{W}_{t}-\frac{\sigma^{2} t}{2}\right\}, \\
& B_{t}=B_{0} \exp \{r t\} .
\end{aligned}
$$

where $\hat{W}_{t}:=W_{t}-\theta t$ is a standard Brownian motion on $\left(\left\{\mathcal{F}_{t}\right\}_{0 \leq t \leq T_{2}}, \hat{P}\right)$, as the Maruyama-Girsanov theorem says.

The fair value at time $t$ of a derivative whose pay-off at time $T$ is $H$, which is the unique one that excludes arbitrage opportunities, is given by

$$
C_{t}=e^{-r(T-t)} \boldsymbol{E}^{\hat{P}}\left[H \mid \mathcal{F}_{t}\right], \quad(0 \leq t \leq T)
$$

the discounted conditional expectation of the pay-off random variable with respect to the EMM.

The hedging strategy for the derivative is obtained via the stochastic differential expression of $C$;

$$
d C_{t}=\eta_{t} d S_{t}+\nu_{t} d B_{t}
$$

which means holding $\eta$ amounts of the asset and $\nu$ amounts of the non-risky asset perfectly hedges the derivative.

For details of the arguments in this section, see e.g. [1] or [2] and the references therein.

\section{Pricing/A Proof of Theorem 1}

In this section, we will give the value of the Exotic Forward following the framework of the previous section.

amount of the bond. 
To apply (13), we unify the cash flows as an $\mathcal{F}_{T_{2}}$ measurable random variable. Let us define an $\mathcal{F}_{T}$ measurable set

$$
\begin{aligned}
& A\left(Y_{T}\right):= \\
& \left\{S_{T_{1}}-K \leq Y_{T}+e^{-r\left(T_{2}-T\right)} E^{\hat{\boldsymbol{P}}}\left[S_{T_{2}}-K-Y_{T} \mid \mathcal{F}_{T}\right]\right\} \\
& =\left\{S_{T_{1}}-K \leq\left(1-e^{-r\left(T_{2}-T\right)}\right) Y_{T}+S_{T}-e^{-r\left(T_{2}-T\right)} K\right\} \\
& =\left\{S_{T_{1}}-S_{T} \leq\left(1-e^{-r\left(T_{2}-T\right)}\right)\left(Y_{T}+K\right)\right\} .
\end{aligned}
$$

On $A\left(Y_{T}\right)$, the buyer is sure to choose $T_{2}$ as the pricedate, while on $\Omega \backslash A\left(Y_{T}\right)$ she/he will not fail to choose $T_{1}$.

The cash flow on $\Omega \backslash A\left(Y_{T}\right)$ is $S_{T_{1}}-K$ at $T$, and will be

$$
e^{r\left(T_{2}-T\right)}\left(S_{T_{1}}-K\right)
$$

at $T_{2}$, if all is invested to the bond. While on $A\left(Y_{T}\right)$, applying the same argument as above, the cash flow at $T_{2}$ is

$$
\begin{aligned}
& e^{r\left(T_{2}-T\right)} Y_{T}+S_{T_{2}}-K-Y_{T} \\
& =\left(e^{r\left(T_{2}-T\right)}-1\right) Y_{T}+S_{T_{2}}-K .
\end{aligned}
$$

Hence, the pay-off at $T_{2}$ of the exotic forward can be seen as

$$
\begin{aligned}
H\left(Y_{T}\right) & =1_{\Omega \backslash A\left(Y_{T}\right)} e^{r\left(T_{2}-T\right)}\left(S_{T_{1}}-K\right) \\
& +1_{A\left(Y_{T}\right)}\left\{\left(e^{r\left(T_{2}-T\right)}-1\right) Y_{T}+S_{T_{2}}-K\right\} .
\end{aligned}
$$

Therefore, applying (13), we have

$$
\begin{aligned}
C_{t}\left(Y_{T}\right) & =e^{-r\left(T_{2}-t\right)} \boldsymbol{E}^{\hat{P}}\left[H\left(Y_{T}\right) \mid \mathcal{F}_{t}\right]\left(0 \leq t \leq T_{2}\right) \\
& =e^{-r\left(T_{2}-t\right)} e^{r\left(T_{2}-T\right)} \boldsymbol{E}^{\hat{P}}\left[1_{\Omega \backslash A\left(Y_{T}\right)}\left(S_{T_{1}}-K\right) \mid \mathcal{F}_{t}\right] \\
& +e^{-r\left(T_{2}-t\right)} \boldsymbol{E}^{\hat{P}}\left[1_{A\left(Y_{T}\right)}\left\{\left(e^{r\left(T_{2}-T\right)}-1\right) Y_{T}\right\} \mid \mathcal{F}_{t}\right] \\
& +e^{-r\left(T_{2}-t\right)} \boldsymbol{E}^{\hat{P}}\left[1_{A\left(Y_{T}\right)}\left(S_{T_{2}}-K\right) \mid \mathcal{F}_{t}\right],
\end{aligned}
$$

and if $t \leq T_{1}$,

$$
\begin{aligned}
& =e^{-r(T-t)} \boldsymbol{E}^{\hat{\boldsymbol{P}}}\left[1_{\Omega \backslash A\left(Y_{T}\right)}\left(S_{T_{1}}-K\right) \mid \mathcal{F}_{t}\right] \\
& +e^{-r(T-t)} \boldsymbol{E}^{\hat{P}}\left[1_{A\left(Y_{T}\right)}\left\{\left(1-e^{-r\left(T_{2}-T\right)}\right) Y_{T}\right\} \mid \mathcal{F}_{t}\right] \\
& +\boldsymbol{E}^{\hat{\boldsymbol{P}}}\left[1 _ { A ( Y _ { T } ) } \left(e^{r t} \boldsymbol{E}^{\hat{P}}\left[e^{-r T_{2}} S_{T_{2}} \mid \mathcal{F}_{T}\right]\right.\right. \\
& \left.\left.\quad-e^{-r\left(T_{2}-t\right)} K\right) \mid \mathcal{F}_{t}\right] \\
& =e^{-r(T-t)}\left(\boldsymbol{E}^{\hat{P}}\left[1_{\Omega \backslash A\left(Y_{T}\right)}\left(S_{T_{1}}-K\right) \mid \mathcal{F}_{t}\right]\right. \\
& +\boldsymbol{E}^{\hat{P}}\left[1 _ { A ( Y _ { T } ) } \left\{\left(1-e^{-r\left(T_{2}-T\right)}\right) Y_{T}\right.\right. \\
& \left.\left.\left.\quad+S_{T}-e^{-r\left(T_{2}-T\right)} K\right\} \mid \mathcal{F}_{t}\right]\right) \\
& =e^{-r(T-t)} \boldsymbol{E}^{\hat{P}}\left[\operatorname { m a x } \left(S_{T_{1}}-K,\right.\right. \\
& \\
& \left.\left.\quad\left(1-e^{-r\left(T_{2}-T\right)}\right) Y_{T}+S_{T}-e^{-r\left(T_{2}-T\right)} K\right) \mid \mathcal{F}_{t}\right] .
\end{aligned}
$$

In particular, we see that

$$
\begin{aligned}
& C_{t}\left(S_{T}-K\right) \\
& \begin{aligned}
=e^{-r(T-t)} \boldsymbol{E}^{\hat{P}}\left[\max \left(S_{T_{1}},\left(2-e^{-r\left(T_{2}-T\right)}\right) S_{T}\right) \mid \mathcal{F}_{t}\right] \\
\quad-e^{-r(T-t)} K,
\end{aligned}
\end{aligned}
$$

and

$$
\begin{aligned}
& \pi=C_{0}\left(S_{T}-K\right) \\
& =e^{-r T} \boldsymbol{E}^{\hat{P}}\left[\max \left(S_{T_{1}},\left(2-e^{-r\left(T_{2}-T\right)}\right) S_{T}\right)\right]-e^{-r T} K .
\end{aligned}
$$

We will calculate the expectation in (22), to complete the proof of Theorem 1 .

The most remarkable property is that $A\left(S_{T}-K\right)$ is independent of $\mathcal{F}_{t}$ for any $t \leq T_{1}$, since

$$
\begin{aligned}
& A\left(S_{T}-K\right) \\
= & \left\{S_{T_{1}}-K \leq\left(2-e^{-r\left(T_{2}-T\right)}\right) S_{T}-K\right\} \\
= & \left\{S_{T_{1}} \leq\left(2-e^{-r\left(T_{2}-T\right)}\right) S_{T}\right\}=A \\
= & \left\{\log S_{0}+\sigma \hat{W}_{T_{1}}+\left(r-\frac{1}{2} \sigma^{2}\right) T_{1} \leq\right. \\
& \left.\log S_{0}+\sigma \hat{W}_{T}+\left(r-\frac{1}{2} \sigma^{2}\right) T+\log \left(2-e^{-r\left(T_{2}-T\right)}\right)\right\} \\
= & \left\{\hat{W}_{T}-\hat{W}_{T_{1}} \geq\right. \\
& \left.\quad-\frac{\left(r-\frac{1}{2} \sigma^{2}\right)\left(T-T_{1}\right)+\log \left(2-e^{-r\left(T_{2}-T\right)}\right)}{\sigma}\right\} \\
= & \left\{\hat{W}_{T}-\hat{W}_{T_{1}} \geq-d_{-} \sqrt{T-T_{1}}\right\} .
\end{aligned}
$$

Therefore,

$$
\begin{aligned}
\pi= & e^{-r T} \boldsymbol{E}^{\hat{\boldsymbol{P}}}\left[S_{T_{1}} ; \Omega \backslash A\right] \\
+ & \left(2-e^{-r\left(T_{2}-T\right)}\right) e^{-r T} \boldsymbol{E}^{\hat{P}}\left[S_{T} ; A\right]-K e^{-r T} \\
= & e^{-r T} \boldsymbol{E}^{\hat{P}}\left[S_{T_{1}}\right]\{\hat{\boldsymbol{P}}(\Omega \backslash A) \\
& \left.\quad+\left(2-e^{-r\left(T_{2}-T\right)}\right) \boldsymbol{E}^{\hat{\boldsymbol{P}}}\left[S_{T} / S_{T_{1}} ; A\right]\right\} \\
& \quad e^{-r\left(T-T_{1}\right)} S_{0}\left\{\Phi\left(-d_{-}\right)\right. \\
& \left.\quad+\left(2-e^{-r\left(T_{2}-T\right)}\right) \boldsymbol{E}^{\hat{\boldsymbol{P}}}\left[S_{T} / S_{T_{1}} ; A\right]\right\} .
\end{aligned}
$$

Noting that

$$
S_{T} / S_{T_{1}}=\exp \left\{\sigma\left(\hat{W}_{T}-\hat{W}_{T_{1}}\right)-\frac{\sigma^{2}}{2}\left(T-T_{1}\right)\right\},
$$

we have

$$
\begin{aligned}
& \boldsymbol{E}^{\hat{\boldsymbol{P}}}\left[S_{T} / S_{T_{1}} ; A\right] \\
& =e^{\left(r-\frac{\sigma^{2}}{2}\right)\left(T-T_{1}\right)} \int_{y \geq-d} \frac{1}{\sqrt{2 \pi}} e^{-\frac{y^{2}}{2}} e^{y \sigma \sqrt{T-T_{1}}} d y \\
& =e^{r\left(T-T_{1}\right)} \int_{y \geq-d} \frac{1}{\sqrt{2 \pi}} e^{-\frac{1}{2}\left(y-\sigma \sqrt{T-T_{1}}\right)^{2}} d y \\
& =e^{r\left(T-T_{1}\right)} \Phi\left(d_{+}\right) .
\end{aligned}
$$

By (24) and (26), we obtain (7).

Remark. For the cases of $Y_{T}=0$, the set $A(0)$ defined in (15) is no longer independent of $\mathcal{F}_{T_{1}}$, and therefore the pricing formula is somehow more complicated than (7). On the other hand, if we have

$$
Y_{T}=c S_{T}-K
$$


for any deterministic $c$, then $A\left(Y_{T}\right)$ is again independent of $\mathcal{F}_{T_{1}}$. Further generalizations of (27) are possible and will be studied elsewhere.

\section{Hedging/A Proof of Theorem 2}

As was mentioned in section 4 , the expression (14) gives the hedging strategy.

Let us start with (19), the expression for all $t \in\left[0, T_{2}\right]$ :

$$
\begin{aligned}
& C_{t} \equiv C_{t}\left(S_{T}-K\right) \\
& =e^{-r(T-t)} \boldsymbol{E}^{\hat{P}}\left[1_{\Omega \backslash A}\left(S_{T_{1}}-K\right) \mid \mathcal{F}_{t}\right] \\
& +e^{-r\left(T_{2}-t\right)} \boldsymbol{E}^{\hat{P}}\left[1_{A}\left\{\left(e^{r\left(T_{2}-T\right)}-1\right)\left(S_{T}-K\right)\right\} \mid \mathcal{F}_{t}\right] \\
& +e^{-r\left(T_{2}-t\right)} \boldsymbol{E}^{\hat{P}}\left[1_{A}\left(S_{T_{2}}-K\right) \mid \mathcal{F}_{t}\right] \\
& =e^{-r(T-t)} \boldsymbol{E}^{\hat{P}}\left[1_{\Omega \backslash A} S_{T_{1}} \mid \mathcal{F}_{t}\right]-e^{-r(T-t)} K \\
& +e^{-r\left(T_{2}-t\right)} \boldsymbol{E}^{\hat{P}}\left[1_{A}\left\{S_{T_{2}}+\left(e^{r\left(T_{2}-T\right)}-1\right) S_{T}\right\} \mid \mathcal{F}_{t}\right] .
\end{aligned}
$$

First, let us consider the case when $t \in\left[T, T_{2}\right)$. Since all terms except $S_{T_{2}}$ are $\mathcal{F}_{t}$ measurable, we have

$$
\begin{aligned}
C_{t} & =e^{-r(T-t)} 1_{\Omega \backslash A} S_{T_{1}}-e^{-r(T-t)} K \\
& +\left(e^{-r(T-t)}-e^{-r\left(T_{2}-t\right)}\right) 1_{A} S_{T} \\
& +1_{A} e^{r t} \boldsymbol{E}^{\hat{P}}\left[e^{-r T_{2}} S_{T_{2}} \mid \mathcal{F}_{t}\right] \\
& =e^{-r(T-t)} 1_{\Omega \backslash A} S_{T_{1}}-e^{-r(T-t)} K \\
& +\left(e^{-r(T-t)}-e^{-r\left(T_{2}-t\right)}\right) 1_{A} S_{T}+1_{A} S_{t} .
\end{aligned}
$$

Therefore, we have

$$
\begin{aligned}
d C_{t}=1_{A} d S_{t}+\left\{1_{A} S_{T}\left(e^{-r T}-e^{-r T_{2}}\right)\right. \\
\left.+1_{\Omega \backslash A} S_{T_{1}} e^{-r T}-e^{-r T} K\right\} d B_{t} .
\end{aligned}
$$

Next, when $t \in\left[T_{1}, T\right)$,

$$
\begin{aligned}
C_{t} & =e^{-r(T-t)} S_{T_{1}} \boldsymbol{E}^{\hat{P}}\left[1_{\Omega \backslash A} \mid \mathcal{F}_{t}\right]-e^{-r(T-t)} K \\
& +e^{-r(T-t)}\left(2-e^{r\left(T_{2}-T\right)}\right) S_{t} \boldsymbol{E}^{\hat{P}}\left[1_{A} S_{T} / S_{t} \mid \mathcal{F}_{t}\right] .
\end{aligned}
$$

Since we have

$$
\begin{aligned}
A= & \left\{S_{T_{1}} \leq\left(2-e^{-r\left(T_{2}-T\right)}\right) S_{T}\right\} \\
= & \left\{S_{T_{1}} / S_{t} \leq\left(2-e^{-r\left(T_{2}-T\right)}\right) S_{T} / S_{t}\right\} \\
= & \left\{-\log \left(S_{T} / S_{t}\right) \leq\right. \\
& \left.\quad \log \left(2-e^{-r\left(T_{2}-T\right)}\right)+\log \left(S_{t} / S_{T_{1}}\right)\right\} \\
= & \left\{\hat{W}_{T}-\hat{W}_{t} \geq\right. \\
- & \left.\frac{\left(r-\frac{1}{2} \sigma^{2}\right)(T-t)+\log \left(2-e^{-r\left(T_{2}-T\right)}\right) S_{t} / S_{T_{1}}}{\sigma}\right\}
\end{aligned}
$$

from (23), the conditional expectations in (31) are explicitly calculated as

$$
\boldsymbol{E}^{\hat{P}}\left[1_{\Omega \backslash A} \mid \mathcal{F}_{t}\right]=\Phi\left(-d_{-}\left(t, S_{t}\right)\right),
$$

and

$$
E^{\hat{P}}\left[1_{A} S_{T} / S_{t} \mid \mathcal{F}_{t}\right]=e^{r(T-t)} \Phi\left(d_{+}\left(t, S_{t}\right)\right) .
$$

Noting that $\Phi\left( \pm d_{ \pm}(t, x)\right) \in C^{1,2}\left(\left[T_{1}, T\right) \times \mathbb{R}\right)$, we can apply Itô's formula to get

$$
\begin{gathered}
d \Phi\left( \pm d_{ \pm}\left(t, S_{t}\right)\right)=\left.\partial_{x} \Phi\left( \pm d_{ \pm}(t, x)\right)\right|_{x=S_{t}} d S_{t} \\
\quad+\left.\left(\partial_{t}+2^{-1} \sigma^{2} x^{2} \partial_{x x}\right) \Phi\left( \pm d_{ \pm}(t, x)\right)\right|_{x=S_{t}} d t \\
= \pm \frac{\phi\left(d_{ \pm}\left(t, S_{t}\right)\right)}{\sigma \sqrt{T-t}}\left[S_{t}^{-1} d S_{t}-\left(r+2^{-1} \sigma^{2} \pm 2^{-1} \sigma^{2}\right)\right] d t .
\end{gathered}
$$

Hence

$$
\begin{aligned}
& d C_{t}=e^{-r T}\left\{S_{T_{1}} \Phi\left(-d_{-}\left(t, S_{t}\right)\right)-K\right\} d B_{t} \\
& +e^{-r T} B_{t} S_{T_{1}} d \Phi\left(-d_{-}\left(t, S_{t}\right)\right) \\
& +\left(2-e^{r\left(T_{2}-t\right)}\right)\left[\Phi\left(d_{+}\left(t, S_{t}\right)\right) d S_{t}+S_{t} d \Phi\left(d_{+}\left(t, S_{t}\right)\right)\right. \\
& \left.\quad+d\left\langle\Phi\left(d_{+}\right), S\right\rangle_{t}\right] \\
& =e^{-r T}\left\{S_{T_{1}} \Phi\left(-d_{-}\left(t, S_{t}\right)\right)-K\right\} d B_{t} \\
& -e^{-r T} B_{t} S_{T_{1}} \frac{\phi\left(d_{-}\left(t, S_{t}\right)\right)}{\sigma \sqrt{T-t}}\left(S_{t}^{-1} d S_{t}-r d t\right) \\
& +\left(2-e^{r\left(T_{2}-t\right)}\right)\left[\Phi\left(d_{+}\left(t, S_{t}\right)\right) d S_{t}+\right. \\
& \quad \frac{S_{t} \phi\left(d_{+}\left(t, S_{t}\right)\right)}{\sigma \sqrt{T-t}}\left\{S_{t}^{-1} d S_{t}-\left(r+\sigma^{2}\right) d t\right\} \\
& \left.\quad+\sigma S_{t} \frac{\phi\left(d_{+}\left(t, S_{t}\right)\right)}{\sqrt{T-t}} d t\right] \\
& =\left[\left(2-e^{r\left(T_{2}-t\right)}\right)\left(\Phi\left(d_{+}\left(t, S_{t}\right)\right)+\frac{\phi(d+}{\sigma \sqrt{T-t}}\right)\right. \\
& \left.+\frac{B_{t} S_{T_{1}} \phi\left(-d_{-}\left(t, S_{t}\right)\right)}{B_{T} S_{t} \sigma \sqrt{T-t}}\right] d S_{t} \\
& +\left[\frac{S_{T_{1}}}{B_{T}}\left(\Phi\left(-d_{-}\left(t, S_{t}\right)\right)+\frac{\phi\left(d-\left(t, S_{t}\right)\right)}{\sigma \sqrt{T-t}}\right)\right. \\
& -\left(2-e^{r\left(T_{2}-t\right)}\right) \frac{S_{t} \phi\left(d_{+}\left(t, S_{t}\right)\right)}{\left.B_{t} \sigma \sqrt{T-t}-\frac{K}{B_{T}}\right] d B_{t}}
\end{aligned}
$$

Finally, consider the cases when $t \in\left[0, T_{1}\right)$. Since $A$ is independent of $\mathcal{F}_{t}$, from (28) we have

$$
\begin{aligned}
C_{t} & =e^{-r\left(T-T_{1}\right)} S_{t} \hat{\boldsymbol{P}}(\Omega \backslash A)-e^{-r(T-t)} K \\
& +e^{-r(T-t)}\left(2-e^{r\left(T_{2}-T\right)}\right) \boldsymbol{E}^{\hat{\boldsymbol{P}}}\left[S_{T_{1}} \mid \mathcal{F}_{t}\right] \boldsymbol{E}^{\hat{\boldsymbol{P}}}\left[1_{A} S_{T} / S_{T_{1}}\right] \\
& =e^{-r\left(T-T_{1}\right)} S_{t} \Phi\left(-d_{-}\right)-e^{-r(T-t)} K \\
& +\left(2-e^{-r\left(T_{2}-T\right)}\right) \Phi\left(d_{+}\right) S_{t} .
\end{aligned}
$$

Therefore,

$$
\begin{aligned}
d C_{t} & =-K e^{-r T} d B_{t}+\left\{e^{-r\left(T-T_{1}\right)} \Phi\left(-d_{-}\right)\right. \\
& \left.+\left(2-e^{-r\left(T_{2}-T\right)}\right) \Phi\left(d_{+}\right)\right\} d S_{t} .
\end{aligned}
$$

By (30), (36) and (38), we get (8) and (9) 


\section{References}

[1] J. Akahori: Stochastic Analysis in Mathematical Finance, (in Japanese) Systems, Control and Information, 44, pp.429-433, 2000.

[2] J. C. Hull Options, Futures, and Other Derivatives, (5th Edition), Prentice Hall, 2002. 